catalectic, while the knot is such that the self-apolar members coincide. The knot then has one form representing both of its possible self-apolar forms and also its three coincident catalectic forms.

This shows that the previous statement is true but that it is a special case of the general situation, derived in this paper, for all types of the curve with an oscnode.

Carnegie Institute of Technology

\title{
THE TRANSFORMATIONS GENERATED BY AN INFINITESIMAL PROJECTIVE TRANSFORMATION IN FUNCTION SPACE*
}

\section{BY I. A. BARNETT}

The problem considered in this note is to determine the one-parameter group of finite transformations generated by an infinitesimal projective transformation in the space of continuous functions. It will be shown that this one-parameter group consists entirely of projective transformations of functionspace in the sense defined by L. L. Dines. $\dagger$ While the problem is completely solved in the paper just cited (p. 57), it will be seen that the result may be obtained directly without the use of the auxiliary formulas developed by Dines in the first part of his paper.

Let the given infinitesimal projective transformation be defined by the integro-differential equation

$$
\begin{gathered}
\frac{\partial \phi^{\prime}(x ; t)}{\partial t}=\lambda(x)+\mu(x) \phi^{\prime}(x ; t)+\int_{0}^{1} \nu(x, y) \phi^{\prime}(y ; t) d y \\
-\phi^{\prime}(x ; t) \int_{0}^{1} \rho(y) \phi^{\prime}(y ; t) d y
\end{gathered}
$$

with the boundary condition $\phi^{\prime}(x ; 0)=\phi(x)$. It is required to

* Presented to the Society, April 6, 1928.

$\dagger$ Projective transformations in function space, Transactions of this Society, vol. 20 (1914), p. 45. 
prove that the unique solution of equation (1), with the given boundary condition, has the form

$$
\phi^{\prime}(x ; t)=\frac{\alpha(x ; t)+\beta(x ; t) \phi(x)+\int_{0}^{1} \gamma(x, y ; t) \phi(y) d y}{\delta(t)+\int_{0}^{1} \epsilon(y ; t) \phi(y) d y},
$$

where the functions $\alpha, \beta, \gamma, \delta$ and $\epsilon$ are to be determined in terms of $\lambda, \mu, \nu$, and $\rho$.

First introduce new variables by means of the transformation*

$$
\Phi(x ; t)=\omega(t) \phi(x ; t), \quad \Phi^{\prime}(x ; t)=\omega^{\prime}(t) \phi^{\prime}(x ; t) .
$$

For $t=0$, these become

$$
\Phi(x)=\omega \phi(x), \quad \Phi^{\prime}(x)=\omega^{\prime} \phi^{\prime}(x),
$$

where

$$
\Phi(x)=\Phi(x ; 0), \quad \omega=\omega(0) .
$$

One readily finds by means of (3) that the single equation (1) may be replaced by the pair of equations

$$
\left\{\begin{array}{l}
\frac{\partial \Phi^{\prime}(x ; t)}{\partial t}=\lambda(x) \omega^{\prime}(t)+\mu(x) \Phi^{\prime}(x ; t)+\int_{0}^{1} \nu(x, y) \Phi^{\prime}(y ; t) d y \\
\frac{\partial \omega^{\prime}(t)}{\partial t}=\int_{0}^{1} \rho(y) \Phi^{\prime}(y ; t) d y
\end{array}\right.
$$

with the boundary conditions $\Phi^{\prime}(x ; 0)=\Phi(x), \quad \omega^{\prime}(0)=\omega$. Likewise, equation (2) goes into the system

* Kowalewski, Sur une classe de transformations infinitésimales, Comptes Rendus, vol. 153 (1911), p. 1452. Kowalewski makes no specific mention of the use of homogeneous coordinates in function space, but the method he gives for reducing the form of an infinitesimal projective transformation to one involving only linear operations is precisely this change of coordinates. In a paper published about eleven years later, Dines actually points out how, by the introduction of such coordinates, he is able to simplify, to a considerable extent, some of the results in his Transactions paper cited above. In this last paper, which is entitled $A$ primary classification of projective transformation in function space (American Journal of Mathematics, vol. 44 (1925)), he suggests the possibility of recasting other results in his Transactions paper. The present note does this for $\$ 9$ of his paper. 


$$
\left\{\begin{aligned}
r \Phi^{\prime}(x ; t) & =\alpha(x ; t) \omega+\beta(x ; t) \Phi(x)+\int_{0}^{1} \gamma(x, y ; t) \Phi(y) d y, \\
r \omega^{\prime}(t) & =\int_{0}^{1} \epsilon(y ; t) \Phi(y) d y+\delta(t) \omega,
\end{aligned}\right.
$$

where $r$ is an arbitrary constant.

The result of differentiating $\left(2^{\prime}\right)$ with respect to $t$ is

$$
\left\{\begin{aligned}
& r \frac{\partial \Phi^{\prime}(x ; t)}{\partial t}=\frac{\partial \alpha(x ; t)}{\partial t} \omega+\frac{\partial \beta(x ; t)}{\partial t} \Phi(x) \\
&+\int_{0}^{1} \frac{\partial \gamma(x, y ; t)}{\partial t} \Phi(y) d y, \\
& r \frac{\partial \omega^{\prime}(t)}{\partial t}=\int_{0}^{1} \frac{\partial \epsilon(y ; t)}{\partial t} \Phi(y) d y+\frac{\partial \delta(\epsilon)}{\partial t} \omega .
\end{aligned}\right.
$$

Substituting $\left(1^{\prime}\right)$ in $(5)$, one finds the relations

$$
\left\{\begin{array}{l}
r\left[\lambda(x) \omega^{\prime}(t)+\mu(x) \Phi^{\prime}(x ; t)+\int_{0}^{1} \nu(x, y) \Phi^{\prime}(y) d y\right] \\
=\omega \frac{\partial \alpha(x ; t)}{\partial t}+\Phi \frac{\partial \beta(x ; t)}{\partial t}+\int_{0}^{1} \frac{\partial \gamma(x, y ; t)}{\partial t} \Phi(y) d y \\
r\left[\int_{0}^{1} \rho(y) \Phi^{\prime}(y ; b) d y\right]=\int_{0}^{1} \frac{\partial \epsilon(y ; t)}{\partial t} \Phi(y) d y+\omega \frac{\partial \delta(t)}{\partial t}
\end{array}\right.
$$

Replacing $\Phi^{\prime}(x ; t)$ and $\omega^{\prime}(t)$ in the left member of (6) by their expressions from $\left(2^{\prime}\right)$, the following equations for the determination of $\alpha, \beta, \gamma, \delta, \epsilon$ are obtained:

$$
\left\{\begin{array}{l}
\omega[\lambda(x) \delta(t)+\mu(x) \alpha(x ; t)]+\Phi(x)[\mu(x) \beta(x ; t)] \\
\quad+\int_{0}^{1} \Phi(y)[\lambda(x) \epsilon(y ; t)+\nu(x, y) \beta(y ; t)+\mu(x) \gamma(x, y ; t) \\
\left.\quad+\int_{0}^{1} \nu(x, z) \gamma(z, y ; t) d z\right] d y=\omega \frac{\partial \alpha(x ; t)}{\partial t}+\Phi(x) \frac{\partial \beta(x ; t)}{\partial t} \\
\quad+\int_{0}^{1} \frac{\partial \epsilon(y ; t)}{\partial t} \Phi(y) d y, \\
\omega \int_{0}^{1} \rho(y) \alpha(y ; t) d y+\int_{0}^{1} \Phi(x)[\rho(x) \beta(x ; t) \\
\left.\quad+\int_{0}^{1} \rho(x) \gamma(x, y ; t) d y\right] d x=\omega \frac{\partial \delta(t)}{\partial t}+\int_{0}^{1} \frac{\partial \epsilon(x ; t)}{\partial t} \Phi(x) d x .
\end{array}\right.
$$


Now it is easy to see that if

$$
\omega A(x)+B(x) \Phi(x)+\int_{0}^{1} C(x, y) \Phi(y) d y
$$

vanishes identically for all real functions $\Phi(x)$ and all real numbers $\omega$, then $A(x) \equiv B(x) \equiv C(x, y) \equiv 0$. Applying this result to equations (7), one finds that the required coefficients of the projective transformations satisfy the following five linear homogeneous integro-differential equations:

$$
\begin{aligned}
\frac{\partial \alpha(x ; t)}{\partial t}= & \mu(x) \alpha(x ; t)+\lambda(x) \delta(t)+\int_{0}^{1} \nu(x, y) \alpha(y ; t) d y, \\
\frac{\partial \beta(x ; t)}{\partial t}= & \mu(x) \beta(x ; t), \\
\frac{\partial \gamma(x, y ; t)}{\partial t}= & \lambda(x) \epsilon(y ; t)+\nu(x, y) \beta(y ; t)+\mu(x) \gamma(x, y ; t) \\
& +\int_{0}^{1} \nu(x, z) \gamma(z, y ; t) d z, \\
\frac{\partial \delta(t)}{\partial t}= & \int_{0}^{1} \rho(y) \alpha(y ; t) d y, \\
\frac{\partial \epsilon(x ; t)}{\partial t}= & \rho(x) \beta(x ; t)+\int_{0}^{1} \rho(y) \gamma(y, x ; t) d y .
\end{aligned}
$$

The initial conditions are

$$
\alpha(x ; 0) \equiv \gamma(x, y ; 0) \equiv \epsilon(x ; 0) \equiv 0 ; \quad \beta(x ; 0) \equiv \delta(0) \equiv 1 .
$$

These are the equations (19) obtained by Dines in the paper already cited, and, as indicated there, their solution may be found as power series in $t$.

The University of Cincinnati 